\title{
Invited comment on the work of Benneker L. et al. "A novel multiple-trauma CT-scanning protocol using patient repositioning may increase risks of iatrogenic injuries"
}

\author{
Hanno Hoppe • Jan Gralla
}

Received: 8 January 2007 / Accepted: 9 January 2007 / Published online: 15 February 2007

(C) Am Soc Emergency Radiol 2007

Dear Editor,

We recently reported a computed tomography (CT)-scanning protocol for chest and abdomen of multiple-trauma patients with upper extremity repositioning to minimize artifacts and enhance image quality [1]. Using this CT protocol, Benneker et al. describe a case of an intubated multipletrauma patient with anterior glenohumeral dislocation and plexus injury, which the authors believe may have been caused by upper extremity repositioning. The authors report that the patient had severe hematoma ventral to the subscapular fossa before the CT scan indicating traumatic shoulder and plexus injury. As clearly stated in our paper, potential fractures and dislocations of the arm and shoulder have to be evaluated clinically before using a CT-scanning protocol with upper extremity repositioning. A hematoma as described by the authors should have been diagnosed clinically before scanning the patient with upper extremity repositioning. If arm or shoulder injury is suspected, CT scanning with upper extremity elevation is contraindicated not to aggravate potential injury, and an alternate CTscanning protocol should be used.

Furthermore, the authors report that, before the CT scan, the patient underwent a conventional full-body X-ray scan in $\mathrm{a}-\mathrm{p}$ and lateral projections to rule out bone injury, but they do not make a statement on if the patient's upper extremities were raised for this scan, which to our knowledge is usually performed. If this was the case, this may have contributed to aggravating the patient's shoulder and plexus injury.
In addition, the authors testify that the patient's anterior glenohumeral dislocation was missed after the CT scan both clinically and on the images, and that it was not diagnosed until the patient had been extubated in the intensive care unit. After extubation, shoulder reduction was performed, but plexus brachialis paresis of the left arm remained. The authors do not comment on the actual duration the patient had glenohumeral dislocation for, but this may have substantially aggravated the patient's injury. Even if anterior glenohumeral dislocation has occurred due to upper extremity repositioning, this should have been diagnosed on the CT scan images, and shoulder reduction should have been performed immediately afterwards to minimize the patient's residual injury.

In conclusion, the reported case does not seem to reflect an actual complication of a CT scan with upper extremity repositioning, but appears to be a series of other mishaps. As clearly stated in our paper, this CT-scanning protocol is only recommended for patients without clinically suspected injury of the arms or shoulders, unlike the reported case. This has to be evaluated clinically before the CT scan, especially in intubated and paralyzed patients, to avoid iatrogenic injury. Used in the correct clinical context, a CTscanning protocol with upper extremity repositioning for scanning chest and abdomen of multiple-trauma patients may substantially contribute to reduce artifacts and enhance image quality.

\section{References}

Department of Radiology, University Hospital Bern, Inselspital, Freiburgstrasse 20,

3010 Bern, Switzerland

e-mail: hoppeh@ohsu.edu
1. Hoppe H, Vock P, Bonel HM, Ozdoba C, Gralla J (2006) A novel multiple-trauma CT-scanning protocol using patient repositioning. Emerg Radiol 13:123-128 